\title{
A brief history of the Italian-Korean symposium on relativistic astrophysics
}

\author{
Hyung Won Lee ${ }^{1} \mathbb{D}$
}

Received: 16 November 2020 / Revised: 4 December 2020 / Accepted: 9 December 2020 / Published online: 16 March 2021

(c) The Korean Physical Society 2021

\begin{abstract}
The series of Italian-Korean Symposia on Relativistic Astrophysics began in 1987, well before the Astrophysics Division of the Korean Physical Society was established in 1995. The symposiums brought together young researchers and scientists of both nations and formed the foundation that would lead to the establishment of the astrophysics division. In this essay, I want to describe a brief history for the series. The series has been ongoing for more than 30 years, thanks to much effort put in by many motivated scientists. The most recent event was in 2019 at Pescara, Italy.
\end{abstract}

Keywords Symposium $\cdot$ Relativistic astrophysics $\cdot$ International collaboration

\section{Introduction}

My first engagement with this series of symposia was in 1987 when I was a Ph.D. student of Sogang university supervised by Prof. Yongduk Kim [1]. At that time, nobody could have predicted that this series would continue for more than 30 years, let alone me still being part of it as I am today.

As all things are, the first symposium was hectic, and nothing seemed to go smoothly. To date, this series of symposia has published about four-hundred articles and has been the center of mass for astrophysicists in Korea for the past 30 years. The subjects of the symposium were changed in step with worldwide trends. Initially, most of the talks were about theoretical general relativity and cosmology. Later, gamma ray bursts (GRBs), dark matter and gravitationalwave-related subjects were introduced. In the following sections, I will describe briefly each of the sixteen symposia.

\section{Early days of the series}

The first symposium was organized by Hanyang University with Prof. Chul H. Lee, H. K. Lee and others. One can find detailed information about this historic symposium in Ref.
[2]. This symposium was held at Hanyang University and Sorak Mountain from September 3 to 8, 1987. It was very successful, and an inauguration ceremony was conducted by Hanyang University and the Italian Embassy. Eighty-three domestic and eight foreign participants attended. The number of domestic participants is strikingly many considering that no astrophysics organization existed in Korea. About sixteen articles were published in World Scientific as a monographs [1].

The second one was held at the University of Rome and Limone Piemonte in Italy from July 8 to 13, 1989. In this symposium, a few young Korean researchers from abroad including K. T. Kim, S. J. Park, and D. La participated. Personally, this was an interesting experience to visit the northern part of Italy, Limone Piemonte. A total of 29 presentations were published in Il Nuovo Cimento [3].

These two symposium are the conerstone that mark the start of the series. Furthermore, these two meetings are the most celebrated gatherings for both Italian and Korean participants as they highlight the first amalgamation of two foreign cultures. While scientific collaboration and exchange of ideas between scientists of two countries is the paramount objective, cultural exchange is another strong motivation for this symposium.

Hyung Won Lee

hwlee@inje.ac.kr

1 College of AI, 197 Inje-ro, Gimhae, South Korea 


\section{Establishing phase}

Most of the symposia were supported by KOSEF (KOrea Science and Engineering Foundation, former organization of the National Research Foundation: NRF) and hosting institutes. Italian participants were supported by the International Center for Relativistic Astrophysics Network (ICRANet) and the Consiglio Nazionale delle Ricerche (CNR). The third symposium was hosted by Ewha Womans University at Gyeongju from July 4 to 8, 1991. The 6th Marcel Grossman Meeting (MG6) was held at Kyoto. Many Italian participants extended their journey to Korea to participate in both MG6 and the Italian-Korean symposium. I also participated in this meeting by extending my trip from Kyoto. A total 25 presented talks were published in the Journal of the Korean Physical Society (JKPS) [4]. Italian participants were very interested in and visited the ancient capital city of Gyeongju and had their first experience with cooked silk worm and were shocked.

The fourth meeting should have been held in Italy in 1993. However, it was postponed to better organize the event, so the fourth symposium was held at 1995 in Italy. This time, it was held in Rome, Gran Sasso and Pescara from July 9 to 15,1995 . Korean participants discussed and decided to maintain the series as a key event of the astrophysics division, and the chief of division became the principal investigator and organizer of this biannual meeting. In return, the Italian partner, Prof. Remo Ruffini, promised to gather more Italian participants. The 34 presentations were published in Il Nuovo Cimento [5].

Sogang University hosted the fifth event at Suanbo from Sep 1 to 6, 1997. Along with Italian participants, French and Chinese scientists participated this time. Twenty-three articles in total were published in the JKPS [6].

The sixth symposium was held at Rome University and ICRANet, at Pescara from July 12 to 24, 1999. It was organized jointly with the third ICRA Network Workshop. Participants were given a chance to visit the southern part of Italy and historic Italian cathedrals. Thirty-nine presentations were published in Il Nuovo Cimento [7].

The seventh gathering was hosted by Inje University on Gangwha Island from July 23 to 28, 2001. The presentations were made in traditional Korean buildings, making it memorable, especially for the Italian participants. Sixteen articles were published in the JKPS [8].

For the eighth symposium, we were back in Pescara, hosted jointly by Rome University and ICRANet, from July 18 to 23, 2003. Eighteen articles were published in Il Nuovo Cimento [9].

The ninth symposium was held at Sejong University on Kumgang Mountain from July 19 to 24, 2005. This symposium was special for both the Korean and the Italian participants, because it was held on Kumgang Mountain in North Korea. It is even more special now because people can no longer visit there. We organized four public lectures, one each by Prof. Don N. Page, Soojong Rey, Li-Zhi Fang and Remo Ruffini. Twenty-five articles were published in the JKPS [10].

\section{Italian-Korean symposium on relativistic astrophysics today}

After the ninth symposium, getting regular funding for the symposium organization became harder. When the event was held in Korea, APCTP mainly supported the event, with the organizing committee providing individual support. In the case of Italy, Korean participants paid for their own travel expenses while local expenses were paid by Italy. With this situation, the number of foreign participants was reduced to around ten, with local participants forming the majority. The tenth symposium was held in ICRANet, in Pescara, from June 25 to 30, 2007. Twenty-four articles were published in the JKPS [11].

The eleventh symposium was held at Sogang University from November 2 to 4, 2009. It was held for a shorter period because it was held during regular semester. Twenty-three articles were published in the JKPS [12]. Next gathering was held hosted by ICRANet, in Pescara, from July 4 to 8, 2011. Twenty-three articles were published in Il Nuovo Cimento [13]. The thirteenth symposium was held at Ewha Womans University from July 15 to 19, 2013. Twenty-seven articles were published in the JKPS [14].

The fourteenth symposium was again held by ICRANet, in Pescara, from July 20 to 24, 2015. This symposium was held just after the fourteenth Marcel Grossman Meeting (MG14) in Rome. The number of Korean participants was reduced, because some could not participate in both events and the proceedings were merged with the proceedings of the MG14. Only nine articles dedicated to the Italian-Korean Symposium were published in the MG14 proceedings [15].

The fifteenth symposium was hosted by Ewha Womans University from July 3 to 7, 2017. It was organized jointly with the 13th International Conference on Gravitation, Astrophysics, and Cosmology (ICGAC-XIII), and 163 scientists participated. Seventy-two articles were published in the EPJ [16].

The latest, the sixteenth meeting, was held by ICRANet, at Pescara, from July 1 to 5, 2019. More than 20 presentations were given and the proceedings are being prepared. The next symposium will be held next year somewhere in Korea. However, we may not be able to organize the meeting beacuse of this COVID-19 pandemic [17], with restrictions still in effect in the year 2021. I hope this series of symposia will 
Table 1 List of Italian-Korean symposia

\begin{tabular}{lllll}
\hline Order & Year & Place & Proceedings & Articles \\
\hline 1 & 1987 & Hanyang University & World Scientific & 16 \\
2 & 1989 & Rome University & Il Nuovo Cimento & 29 \\
3 & 1991 & Ewha Womans University & JKPS & 25 \\
4 & 1995 & Rome University & Il Nuovo Cimento & 34 \\
5 & 1997 & Sogang University & JKPS & 23 \\
6 & 1999 & Rome University & Il Nuovo Cimento & 39 \\
7 & 2001 & Inje University & JKPS & 16 \\
8 & 2003 & Rome University & Il Nuovo Cimento & 18 \\
9 & 2005 & Sejong University & JKPS & 25 \\
10 & 2007 & ICRANet, Pescara & JKPS & 24 \\
11 & 2009 & Sogang University & JKPS & 23 \\
12 & 2011 & ICRANet, Pescara & Il Nuovo Cimento & 23 \\
13 & 2013 & Ewha Womans University & JKPS & 27 \\
14 & 2015 & ICRANet, Pescara & MG14, World Scientific & 9 \\
15 & 2017 & Ewha Womans University & EPJ Web Conf. & 72 \\
16 & 2019 & ICRANet, Pescara & Not Yet & \\
\hline
\end{tabular}

be continued to encourage scientific interactions between our two countries especially for our researchers.

\section{Summary}

The series of "Italian-Korean Symposium on Relativistic Astrophysics" has a rather long history of 32 years, which is rich with fascinating subjects and noteworthy articles. Table 1 represents a list of all Italian-Korean symposia held up to date. While symposium is definitely a positive addition for Astrophysics Division in the Korean Physical Society, it is currently facing hardships that call for careful nurture. Today, the series of symposia is being maintained by a handfull of physicists in Astrophysics Division the Korean Physical Society and Italian partners at ICRANet. If this historic collaboration between Italy and Korea is to be sustained, participation from both Italy and Korea must be actively encouraged, which should ultimately inspiring young, aspiring scientists and those with inquisitive minds to consider working on this field.

Acknowledgements The author thanks the NRF for its continuous support for this series of symposia, the APCTP; Korean institutes such as Hanyang University, Sogang University, Ewha Womans University, Sejong University, Yonsei University, and Inje University; and Italian Institutes, such as the Italian Embassy in Seoul, ICRANet, CNR and Ministero degli Affari Esteri e delle Cooperazione Internazionale (MAE) for their support. The author was supported by the National Research Foundation of Korea (NRF) (No. NRF-2018R1D1A1B05049338).

\section{References}

1. Y.D. Kim, C.H. Lee, R. Ruffini (eds.), 1st Italian-Korean Symposium on Relativistic Astrophysics (World Scientific, Singapore, 1987)

2. History of 50 years of the Korean Physical Society (in Korean). http://www. kps.or.kr/content/50years/html/main.htm

3. Y.D. Kim, R. Ruffini (eds.), Il Nuovo Cimento B 105, 817-1078 (1990)

4. Y.D. Kim, J. Yang, S.-W. Kim (ed.), J. Korean Phys. Soc. 25, S179-S309 (1992)

5. V. Coco, R. Ruffini (eds.), Il Nuovo Cimento B 112, 139-529 (1995)

6. S.-W. Kim, H.W. Lee (eds.), J. Korean Phys. Soc. 33, S471-S598 (1998)

7. C. Cherubini, R. Ruffini (eds.), Il Nuovo Cimento B 115, $697-$ 1137 (1999)

8. H.W. Lee (ed.), J. Korean Phys. Soc. 42, S1-S112 (2003)

9. C. Cherubini, H.W. Lee (eds.), Il Nuovo Cimento B 120, 1021$1242(2005)$

10. Y.W. Kang, H.W. Lee (eds.), J. Korean Phys. Soc. 49, 697-844 (2006)

11. R. Ruffini, S.-W. Kim (eds.), J. Korean Phys. Soc. 56, 1575-1699 (2010)

12. S.P. Kim, B.H. Lee (eds.), J. Korean Phys. Soc. 57, 551-679 (2010)

13. H.W. Lee, R. Ruffini, G. Vereshchagin (eds.), Il Nuovo Cimento C 36, 10269 (2013)

14. S.-W. Kim (ed.), J. Korean Phys. Soc. 65, 801-951 (2014)

15. R. Ruffini (ed.), Proceedings of MG14 (World Scientific, 2017), pp. 4375-4439

16. B. Gwak, G. Kang, C. Kim, H.C. Kim, C.H. Lee, J. Lee, S. Lee, W. Lee (eds.), EPJ Web Conf., vol. 168 (2018)

17. https://en.wikipedia.org/wiki/COVID-19_pandemic

Publisher's Note Springer Nature remains neutral with regard to jurisdictional claims in published maps and institutional affiliations. 